\title{
Efeito de Agentes Térmicos Aplicados Previamente a um Programa de Alongamentos na Flexibilidade dos Músculos Isquiotibiais Encurtados
}

\author{
Effect of Thermal Agents Previously Applied to a Program of Prolongations \\ in the Flexibility of the Shortened Isquiotibial Muscles
}

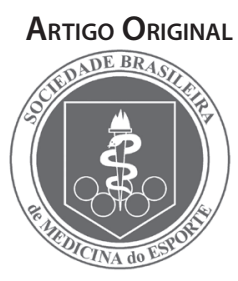

Luis Ulisses Signori

Fernando Roberto Schütz Voloski ${ }^{1}$

Alessandra Cristina Kerkhoff ${ }^{1}$

Léia Brignoni'

Rodrigo Della Méa Plentz

1. Universidade de Cruz Alta / UNICRUZ / Departamento de Fisioterapia

\section{Endereço para correspondência: \\ Fundação Universidade de Cruz Alta - UNICRUZ \\ Unidade de Pesquisa - Dr. Luis \\ Ulisses Signori \\ Av. General Osório, 1.323, Centro \\ 98005-150 - Cruz Alta, RS. \\ Tel.: (55) 3322-2962 \\ Fax: (55) 3321-1513 \\ E-mail:I signori@universiabrasil.net}

Submetido em 13/02/2007

Versão final recebida em 26/12/2007

Aceito em 04/01/2008

\begin{abstract}
RESUMO
A hipotermia e a hipertermia são sugeridas como meios físicos para aumentar a flexibilidade. O objetivo deste estudo foi comparar o efeito desses agentes térmicos aplicados previamente a um programa de alongamentos dos músculos isquiotibiais (ITs) encurtados. Foram selecionados 42 voluntários saudáveis com encurtamento de ITs avaliados pelo teste do Banco de Wells e Dillon (BWD). A amostra foi aleatoriamente dividida em quatro grupos: G1 (controle), n: 10; sete masculinos (M); $23 \pm 0,8$ anos (a); $23,4 \pm 0,7 \mathrm{~kg} / \mathrm{m}^{2} ; \mathrm{G} 2$ (hipo+alongamentos), n: 12; 9 M; $23 \pm 1,2 a ; 22,8 \pm 0,6 \mathrm{~kg} / \mathrm{m}^{2}$, G3 (hiperalongamentos), n: 12; $10 \mathrm{M} ; 21,5 \pm$ 0,5a; $23,17 \pm 0 ; 6 \mathrm{~kg} / \mathrm{m}^{2 ;}$ e G4 (alongamentos), n: $8 ; 6 \mathrm{M} ; 24,7 \pm 1,7 \mathrm{a} ; 23,2 \pm 0,4 \mathrm{~kg} / \mathrm{m}^{2}$. Os grupos G2, G3 e G4 foram submetidos a um programa de alongamentos estáticos, consistindo de 13 sessões (15 min cada) e seguimento de 21 dias. Cada sessão compreendeu uma série de 15 exercícios para cada membro inferior; o tempo de manutenção de cada alongamento foi de 30s e igual tempo de intervalo. Os agentes térmicos foram aplicados através de bolsas térmicas (diâmetro de $30 \mathrm{~cm}$ ) por 15 min, precedendo os alongamentos dos ITs. A temperatura foi controlada para o $\mathrm{G} 2$ entre $1{ }^{\circ} \mathrm{e} 4^{\circ} \mathrm{C}$ e, para o $\mathrm{G} 3$, de $41^{\circ}$ a $45^{\circ} \mathrm{C}$. Observou-se que os grupos G2, G3 e G4 apresentaram aumento quantitativo na variação da flexibilidade dos ITs (BWD: G2: 10,8 $\pm 1,2$; G3: $11,5 \pm 1,4 ; G 4: 11,07 \pm 1,7 \mathrm{~cm} ; P<0,01)$ em relação ao grupo controle (G1: $-0,14 \mathrm{~cm})$. Não foram observadas diferenças entre os grupos que aumentaram a flexibilidade. Conclui-se que o aumento da flexibilidade se deve aos alongamentos, e independe da aplicação prévia de hipertermia e/ou hipotermia por condução.
\end{abstract}

Palavras-chave: hipotermia, hipertermia, flexibilidade, exercícios de alongamento muscular.

\begin{abstract}
The hypothermia and hyperthermia are suggested as physical means to increase the flexibility. The objective of this study was to compare the effect of these thermal agents applied previously to a program of muscles stretching isquiotibiais (ITs) shortened. 42 healthy volunteers were selected with shortening of appraised ITs by the sit-and-reach test (BWD). The sample was random divided in 4 groups: G1 (controls) n:10; 7 masculine (M); 23 $\pm 0,8$ years (y); $23,4 \pm 0,7 \mathrm{~kg} / \mathrm{m}^{2}, \mathrm{G} 2$ (hypo+stretching) $\mathrm{n}: 12 ; 9 \mathrm{M} ; 23 \pm 1,2 \mathrm{y} ; 22,8 \pm 0,6 \mathrm{~kg} / \mathrm{m}^{2}, \mathrm{G} 3$ (hyper+stretching) $\mathrm{n}: 12 ; 10 \mathrm{M} ; 21,5 \pm 0,5 \mathrm{y} ; 23,17 \pm 0 ; 6 \mathrm{~kg} / \mathrm{m}^{2}$ and G4 (stretching) $\mathrm{n}: 8 ; 6 \mathrm{M} ; 24,7 \pm 1,7 \mathrm{y} ; 23,2 \pm 0,4 \mathrm{~kg} / \mathrm{m}^{2}$. The groups $\mathrm{G} 2$, G3 and G4 were submitted to the program of static prolongations, consisting of 13 sessions (15 min each) and continuation within 21 days. Each session consisted of a series of 15 exercises for each inferior member, and the time of maintenance of each prolongation was of 30 s and equal time of interval. The thermal agents were applied through thermal bags (diameter of $30 \mathrm{~cm}$ ) for $15 \mathrm{~min}$, preceding the prolongations of ITs, the temperature was controlled for $\mathrm{G} 2$ between $1{ }^{\circ}$ to $4^{\circ} \mathrm{C}$ and $\mathrm{G} 341^{\circ}$ to $45^{\circ} \mathrm{C}$. It was observed that the groups $\mathrm{G} 2$, G3 and G4 presented quantitative in the variation of the flexibility of ITs (BWD: G2: 10,8 $\pm 1,2 ; \mathrm{G} 3: 11,5 \pm 1,4$; G4: $11,07 \pm 1,7 \mathrm{~cm} ; P<0,01)$ in relation to the control group $(\mathrm{G} 1:-0,14 \mathrm{~cm})$. Differences were not observed among the groups that increased the flexibility. In conclusion the increase of the flexibility is due to stretching, and this does not depend on the previous application of hyperthermia and/or hypothermia for transport.
\end{abstract}

Keywords: hypothermia, hyperthermia, flexibility, muscle stretching exercises.

\section{INTRODUÇÃO}

Flexibilidade adequada é uma característica importante de aptidão física, sendo um componente fundamental para a qualidade de vida ${ }^{(1)}$, performance esportiva, prevenção e reabilitação de lesões ${ }^{(2)}$. Exercícios com finalidade de aumentar a força e a capacidade aeróbica diminuem a flexibilidade muscular e este efeito pode levar a risco aumentado de lesões durante os treinamentos, competições e/ou participação em atividades físicas diárias (2).
Baseados nessa suposição, atletas e desportistas dedicam muitas horas de treinamento para o desenvolvimento e/ou a manutenção de flexibilidade, principalmente pelo uso de vários métodos de alongamento ${ }^{(3)}$. São usadas várias metodologias para aumentar a flexibilidade, dentre estas, as técnicas de Facilitação Neuromuscular Proprioceptiva (FNP), os métodos de alongamento passivo, estático e balístico. Entretanto, o método mais freqüentemente empregado é o alongamento estático; isso ocorre 
devido a sua fácil aplicação e controle de suas variáveis ${ }^{(4)}$. Alongamento estático é executado colocando-se os músculos a serem alongados ao maior comprimento possível e mantendo-os nessa condição durante um período de tempo, posteriormente seguido de intervalo(5). Alongamento estático resulta na diminuição da resistência muscular passiva, devido ao aumento na viscosidade e elasticidade da unidade musculotendínea, resultando no aumento do comprimento muscular ${ }^{(6-7)}$.

O uso prévio da termoterapia em programas de alongamentos é sugerido por proporcionar melhores resultados na flexibilidade e diminuir o desconforto durante as sessões de alongamento ${ }^{(8)}$. Dentre as técnicas de termoterapia, destacam-se a hipotermia (hipo) e a hipertermia (hiper), sendo geralmente aplicadas através de meios como a água, géis e/ou as bolsas térmicas em diferentes temperaturas, como fontes de condução e modulação do nível de calor desejado. O uso da hipotermia associada aos alongamentos pode promover maior efeito na flexibilidade muscular ${ }^{(3)}$; em oposto a esse efeito térmico, a hipertermia também é sugerida em conjunto com os exercícios de flexibilidade ${ }^{(9-10)}$. Burke et al. (2001) sugerem que em indivíduos saudáveis não ocorrem diferenças entre os agentes térmicos aplicados frente a um protocolo de alongamentos ${ }^{(11)}$.

A implementação clínica do calor é sugerida por aumentar a extensibilidade das fibras do colágeno, favorecendo o relaxamento das propriedades mecânicas do músculo(10). Por outro lado, a hipotermia induz a redução do reflexo de estiramento, redução do espasmo muscular, diminuição da inflamação e da sensibilidade dolorosa durante os alongamentos, resultando assim em maior flexibilidade ${ }^{(3,12)}$. Apesar do conhecimento fisiológico do efeito desses agentes térmicos, ainda não há um consenso quanto a sua aplicação prévia sobre um programa de alongamentos. O objetivo deste estudo foi verificar se o emprego de agentes térmicos por condução aplicados previamente a um programa de alongamentos aumenta o ganho na flexibilidade dos músculos isquiotibiais encurtados.

\section{MÉTODOS}

A pesquisa foi realizada entre novembro de 2005 e abril de 2006 e, os dados coletados no Laboratório de Fisiologia do Exercício da Universidade de Cruz Alta (UNICRUZ). Todos os voluntários assinaram o termo de consentimento, conforme resolução 196/96 do Conselho Nacional de Saúde para experimentos com humanos.

Inicialmente, os voluntários foram submetidos a uma avaliação física funcional para identificar os critérios de inclusão e exclusão. Os critérios de inclusão foram: idade entre 18 e 34 anos que apresentavam encurtamento de músculos isquiotibiais (ITs), verificado pelo exame clínico e pelo teste do Banco de Wells e Dillon ${ }^{(13-14), ~ e ~ q u e ~ n a ̃ o ~ p o s s u i ́ a m ~}$ nenhuma patologia crônica, estando em boas condições de saúde. E como critérios de exclusão: voluntários que apresentassem IMC > 30 $\left(\mathrm{kg} / \mathrm{m}^{2}\right)$; cirurgias prévias de coluna e/ou membros inferiores; presença de processo inflamatório musculoesquelético; relato prévio de crises de lombalgia e lombociatalgia; testes de radiculite positivos (teste da perna retificada e teste de Laségue), estiramento ou contratura muscular em período inferior a 90 dias ao início do programa; realização de atividades complementares de alongamentos (loga, reeducação postural global, capoeira). Para as medidas físicas do peso e da altura utilizou-se uma balança antropométrica com estadiômetro de marca Welmy (modelo 110).

A amostra foi composta de forma aleatória por 42 voluntários, com idade entre 18 e 34 anos, de ambos os gêneros, divididos em quatros grupos: grupo controle (G1), com dez indivíduos que não foram submetidos a nenhum tipo de intervenção, sendo apenas avaliada a flexibilidade dos músculos isquiotibiais; grupo hipo+alongamentos (G2), com 12 voluntários; grupo hiper+alongamentos (G3), com 12 voluntários; e grupo alongamentos (G4), com oito voluntários.

\section{a) Hipotermia}

A hipotermia foi aplicada por meio de duas bolsas de borracha (diâmetro de $30 \mathrm{~cm}$ ) contendo água fria, situando-se a temperatura entre $1^{\circ} \mathrm{e}$ $4^{\circ} \mathrm{C}$, em forma de compressa por condução, colocada diretamente sobre os músculos isquiotibiais de ambos os membros inferiores, com tempo de aplicação de 15 minutos, antecedendo ao programa de alongamentos.

\section{b) Hipertermia}

A hipertermia foi realizada por meio de duas bolsas de borracha (diâmetro de $30 \mathrm{~cm}$ ) contendo água quente, situando-se a temperatura entre $41^{\circ}$ e $45^{\circ} \mathrm{C}$, em forma de compressa por condução, colocada diretamente sobre os músculos isquiotibiais de ambos os membros inferiores, com tempo de aplicação de 15 minutos, antecedendo ao programa de alongamentos.

\section{c) Alongamentos}

O tipo de alongamento aplicado foi o estático mantido, sendo este efetuado por pesquisador treinado e aplicado de igual forma para os grupos G2, G3 e G4. Os voluntários ficavam deitados em tatame, na posição de decúbito dorsal ;, individualmente, cada joelho era estendido ao ponto de desconforto (tolerância à extensão) com o objetivo de produzir relaxamento e alongamento dos músculos isquiotibiais de ambos os membros inferiores. Cada sessão de 15 minutos consistia em uma série de 15 alongamentos para cada segmento. Cada alongamento foi aplicado com duração de estímulo (ponto de desconforto) de 30 segundos, seguido de igual tempo de intervalo.

\section{MENSURAÇÃO DA FLEXIBILIDADE}

A flexibilidade foi avaliada pelo teste de Wells e Dillon, também denominado de teste de "sentar e alcançar" (sit-and-reach test), que é uma medida linear e quantitativa ${ }^{(13)}$ e que consiste em mensurar a distância em centímetros em relação ao ponto zero, situado ao nível da região plantar. O indivíduo permanece sentado no chão, com os joelhos estendidos, e flexiona o tronco com os membros superiores estendidos, registrando-se ai o maior valor alcançado ao final do movimento. Os valores são expressos em centímetros $(\mathrm{cm})$, sendo o ponto zero $(0 \mathrm{~cm})$ quando as mãos chegam ao nível da região plantar. Os valores positivos correspondem à localização dos dedos das mãos quando ultrapassam a região plantar; são considerados valores negativos quando a posição das mãos não atinge esse ponto ${ }^{(14)}$. A flexibilidade foi medida individualmente no início de cada sessão e os dados foram expressos pelo maior valor das três medidas repetidas.

O teste envolve uma flexão lenta, gradual das regiões lombares e torácicas. Ocorrem também ântero-versão pélvica, elevação da escapular, rotação, flexão e adução horizontal dos ombros, extensão dos cotovelos, punhos e dedos. Isso acarreta envolvimento dos músculos eretores da coluna, glúteos e gastrocnêmicos ${ }^{(15)}$.

\section{PROCEDIMENTOS}

O protocolo experimental consistia em 13 sessões, realizadas em três semanas, não havendo intervalo superior a 48 horas entre cada intervenção. Os procedimentos foram realizados entre as 17 e 20 horas, em temperatura ambiente controlada entre $22^{\circ}$ e $26^{\circ} \mathrm{C}$. A flexibilidade dos voluntários submetidos ao programa de alongamentos nos grupos G2 (hipo+alongamentos), G3 (hiper+alongamentos) e G4 (alongamentos) foi realizada previamente a cada sessão; essas avaliações consistiam em três medidas do teste. No G1 (controle) realizaram-se apenas as avaliações da flexibilidade. Nos grupos G2 e G3, anteriormente à realização dos exercícios de alongamentos, foram utilizados meios físicos de condução (hipotermia e hipertermia, respectivamente) durante 15 minutos, enquanto que o G4 não fez uso de meios físicos de condução, apenas os alongamentos. 


\section{ANÁLISE ESTATÍSTICA}

Os dados estão apresentados em forma de média e erro padrão. A distribuição do gênero foi avaliada pelo teste do qui-quadrado $\left(\chi^{2}\right)$. Para as variáveis contínuas intragrupo foi aplicado o teste pareado de Student. Os dados contínuos entre os grupos foram analisados pela ANOVA fator único, seguido do post hoc de Tukey, sendo considerado significante $P \leq 0,05$.

\section{RESULTADOS}

A caracterização dos grupos que participaram do presente estudo encontra-se na tabela 1. Observa-se que a distribuição dos grupos, em relação ao gênero, apresenta preponderância de aproximadamente ( ) $70 \%$ dos voluntários masculinos. Em relação à idade, o peso e a altura não apresentam diferenças. O IMC inicial e o final não apresentaram diferenças entre os grupos, encontrando-se dentro de um biótipo de voluntários normais. Salienta-se que todos os voluntários apresentavam valores negativos na medida da flexibilidade, avaliada pelo Banco de Wells e Dillon.

Tabela 1. Caracterização dos grupos

\begin{tabular}{l|c|c|c|c|c}
\hline \multicolumn{1}{c|}{ Variáveis } & G1 & G2 & G3 & G4 & $P$ \\
\hline Gênero & $7 \mathrm{M} / 3 \mathrm{~F}$ & $9 \mathrm{M} / 4 \mathrm{~F}$ & $10 \mathrm{M} / 2 \mathrm{~F}$ & $7 \mathrm{M} / 3 \mathrm{~F}$ & 0,960 \\
\hline Idade (anos) & $23 \pm 0,8$ & $23 \pm 1,2$ & $21,5 \pm 0,5$ & $24,75 \pm 1,7$ & 0,274 \\
\hline Peso inicial $(\mathrm{kg})$ & $74,5 \pm 3,9$ & $69,5 \pm 3$ & $72,93 \pm 2,8$ & $72,88 \pm 4,5$ & 0,547 \\
\hline Altura inicial $(\mathrm{m})$ & $1,7 \pm 0,02$ & $1,72 \pm 0,01$ & $1,77 \pm 0,01$ & $1,77 \pm 0,03$ & 0,286 \\
\hline IMC inicial $\left(\mathrm{kg} / \mathrm{m}^{2}\right)$ & $23,4 \pm 0,7$ & $22,8 \pm 0,6$ & $23,17 \pm 0,6$ & $23,23 \pm 0,6$ & 0,937 \\
\hline IMC final $\left(\mathrm{kg} / \mathrm{m}^{2}\right)$ & $22,9 \pm 0,7$ & $23,7 \pm 0,6$ & $23,02 \pm 0,7$ & $22,08 \pm 0,5$ & 0,634 \\
\hline BWD inicial $(\mathrm{cm})$ & $-5,3 \pm 1,2$ & $-3,9 \pm 1,1$ & $-3,1 \pm 1,3$ & $-5,5 \pm 1,1$ & 0,351 \\
\hline
\end{tabular}

IMC: Índice de massa corporal, BWD: Banco de Wells e Dillon

Os voluntários não referiram dor ou desconforto após cada sessão e/ou durante o programa de alongamentos. O grupo controle não apresentou variação da flexibilidade durante o período de acompanhamento. Entretanto, os grupos G1, G2 e G3 apresentaram alterações significantes intragrupos (inicial vs final) na flexibilidade $(P<0,01)$ no final do estudo (13 sessões realizadas em 21 dias). Dados apresentados na figura 1.

Figura 1. Comparações da flexibilidade intragrupo

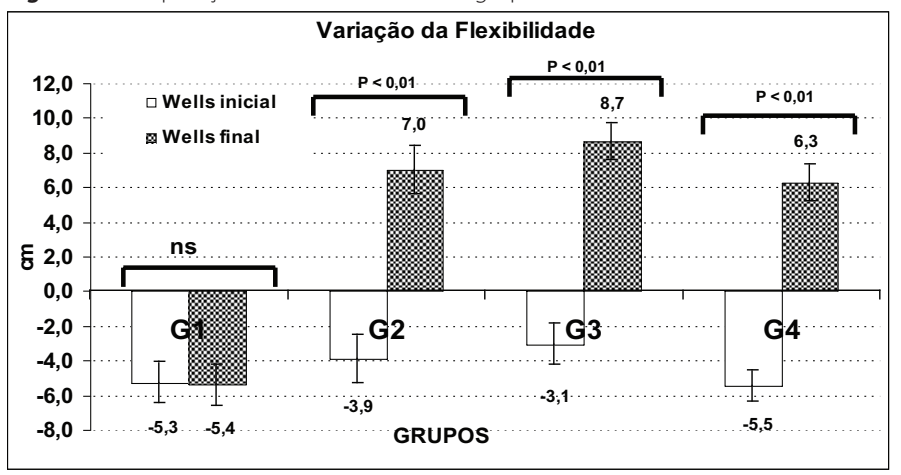

NS: Não significativo.

A comparação de resultados de flexibilidade entre os grupos não demonstrou diferença da avaliação inicial. Entretanto, apresentou significativo aumento após o programa de treinamento $(P<0,01)$, constatando-se, também, que as alterações não mostraram diferenças entre os grupos G2, G3 e G4 (tabela 2). O delta de variação para esses grupos foi de aproximadamente $11 \mathrm{~cm}$.
Tabela 2. Alterações da flexibilidade nos grupos

\begin{tabular}{l|c|c|c|c|c}
\hline \multicolumn{1}{c|}{ Flexibilidade } & G1 & G2 & G3 & G4 & $P$ \\
\hline BWD final $(\mathrm{cm})$ & $-5,4 \pm 1,2$ & $* 6,9 \pm 1,4$ & $* 8,8 \pm 1,7$ & $* 6,2 \pm 0,9$ & $<0,01$ \\
\hline Delta de variação $(\mathrm{cm})$ & $-0,1 \pm 0,6$ & $* 10,8 \pm 1,2$ & $* 11,5 \pm 1,4$ & $* 11,07 \pm 1,7$ & $<0,01$ \\
\hline
\end{tabular}

BWD: Banco de Wells e Dillon; post hoc de Tukey: $P \leq 0,05$.

Em relação às sessões, observa-se que, a partir do quarto dia, nos grupos G2 (0,4cm), G3 (1,7cm) e G4 (0,58cm) os valores médios passam a ser positivos (figura 2) e, que ao final do protocolo, os voluntários não apresentavam encurtamento muscular. O aumento médio na flexibilidade em cada sessão foi de $0,83 \mathrm{~cm}$ para o $\mathrm{G} 2$, de $0,88 \mathrm{~cm}$ para $\mathrm{G} 3$ e de $0,85 \mathrm{~cm}$ para o $\mathrm{G} 4$.

Figura 2. Comportamento da flexibilidade em relação ao número de sessões

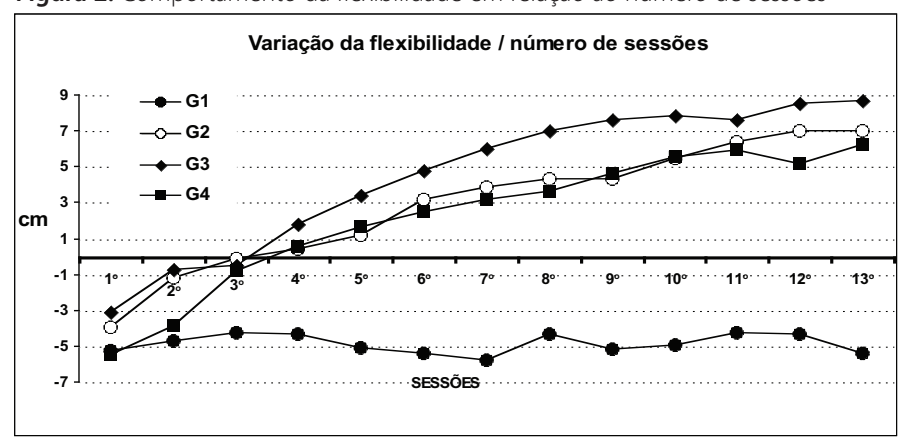

\section{DISCUSSÃO}

Como esperado, o programa de alongamentos proporcionou aumento na flexibilidade dos músculos isquiotibiais encurtados, nos grupos tratados. Os meios físicos de hipotermia e hipertermia na forma de condução não influenciaram o aumento da flexibilidade desses músculos, quando aplicados previamente a um programa de alongamentos por três semanas.

O aumento na flexibilidade deve-se ao remodelamento dos tecidos conjuntivo, muscular (endomísio, perimísio e epimísio) e tendíneo, onde as mudanças no comprimento dos tecidos podem ser quantitativas e organizacionais(16). Essas modificações são acompanhadas por adaptações nas proteínas de miocontráteis do citoesqueleto dos sarcômeros ${ }^{(17)}$. O resultado dessas alterações leva a remodelamento das unidades musculotendínosas, as quais são acompanhadas do aumento da extensibilidade, no comprimento e na resistência ao movimento passivo dinâmico ${ }^{(17)}$. Os alongamentos também são sugeridos para prevenção das lesões musculotendinosas ${ }^{(19)}$. Entretanto, ainda não existem evidências científicas que suportem a afirmação que os alongamentos realizados antes e/ou após exercícios previnem lesões esportivas, mas estes não são desaconselháveis ${ }^{(20)}$. Porém, é inegável a associação da flexibilidade e da atividade física com a melhora da qualidade de vida(1).

A implementação clínica do calor é sugerida por aumentar a extensibilidade das fibras do colágeno e as propriedades mecânicas do músculo(10). Nossos resultados indicam que a aplicação prévia de hipertermia por condução não proporciona benefícios na flexibilidade de músculos encurtados, colaborando com estudos prévios ${ }^{(9,11)}$. Salienta-se que o uso do alongamento associado ao calor (por condução) mostrou-se ineficiente para a prevenção das lesões musculares de jovens submetidos a programa de exercícios de alta intensidade(21). Entretanto, estudos 
experimentais em animais (ratos) comparando diferentes modalidades de hipertermia (Infravermelho e ultra-som) associada a seis sessões de alongamentos sugerem que essa associação seja mais efetiva que os alongamentos para aumentar a extensibilidade de músculos moderadamente contraídos. Outra forma de indução de hipertermia estudada em conjunto com os alongamentos tem sido a diatermia (ondas curtas), onde resultados favoráveis também foram observados na melhora da flexibilidade(22). A discordância dos resultados nesses estudos deve-se possivelmente à forma de indução da hipertermia realizada por irradiação e por conversão nos estudos apresentados, enquanto que, em nossa pesquisa, foi utilizado o método de condução.

A hipotermia associada a alongamentos é sugerida como mais eficiente que o aquecimento prévio aos alongamentos e/ou apenas os alongamentos ${ }^{(3,12)}$. Os autores sugerem que a hipotermia associada aos alongamentosfisiologicamente induzem a redução do reflexo de estiramento, redução do espasmo muscular, diminuição da inflamação e da sensibilidade dolorosa durante os alongamentos, resultando, assim, em maior flexibilidade. Outro fato a ser considerado é que a hipotermia favorece a diminuição da temperatura muscular pós-exercício, podendo colaborar de forma indireta para o aumento da flexibilidade ${ }^{(23)}$. Entretanto, nossos resultados não confirmam essa afirmação, em razão de o efeito agudo na flexibilidade pós-sessão de alongamentos resultar em maior adaptação fisiológica apenas durante a avaliação e não promover resultado favorável durante programas de alongamentos crônicos. Um único alongamento dos músculos isquiotibias, com duração de 90s, resulta em redução de 30\% na viscoelasticidade; entretanto, esse efeito dura por apenas uma hora após o término do alongamento(6). Estudos de Taylor et al. (1995)(9) e Burke et al. (2001)(11) mostraram que a hipotermia não favorece o aumento da flexibilidade após programa de alongamentos, confirmando nossos resultados.

O programa de alongamento a longo prazo (três semanas), empregado neste estudo, promoveu o aumento da flexibilidade; o ga- nho na amplitude de movimento articular deve-se provavelmente às mudanças nas propriedades musculotendíneas ${ }^{(6)}$. Entretanto, deve-se destacar que os resultados de um programa de alongamentos na flexibilidade sofrem a influência do tipo de alongamento, do número de séries, da duração de cada alongamento no ponto de desconforto e do momento da avaliação(24), os quais não são objeto de discussão neste artigo.

Apesar de ser amplamente utilizado para a avaliação da flexibilidade, o Banco de Wells e Dillon ${ }^{(13-14)}$ não avalia apenas os músculos isquiotibiais, mas também a musculatura do membro superior, posterior do tronco e membros inferiores ${ }^{(15)}$, podendo ser esta uma limitação do estudo. Entretanto, há de se destacar que o exercício selecionado para o programa de alongamento foi direcionado aos isquiotibiais e que os demais músculos sofrem pouca influência deste exercício. Em relação à aplicação dos agentes térmicos apenas no local dos isquiotibiais, não acreditamos que afete os resultados, pois em estudo semelhante onde a fonte térmica era a imersão na água até a região glútea por 10 minutos, os agentes térmicos não interferiram na flexibilidade muscular ${ }^{(11)}$. Outra variável que pode ter influenciado nos resultados é a presença do gênero feminino nos grupos, pois se sabe que as mulheres, geralmente, são mais flexíveis que os homens ${ }^{(25)}$, informação confirmada em nosso estudo, embora a maioria dos voluntários com encurtamento dos isquiotibiais nos grupos estudados fossem homens; saliente-se que a distribuição das mulheres nos grupos foi homogênea.

Concluímos, a partir das informações obtidas neste estud,o que a aplicação prévia de agentes térmicos por condução não potencializa o efeito do alongamento no ganho da flexibilidade dos músculos isquiotibiais encurtados.

Todos os autores declararam não haver qualquer potencial conflito de interesses referente a este artigo.

\section{REFERÊNCIAS BIBLIOGRÁFICAS}

1. Kell RT, Bell G, Quinney A. Musculoskeletal fitness, health outcomes and quality of life. Sports Med 2001;31:863-73.

2. Holt LE, Holt JB, Pelham TW. What research tells us about flexibility I and II. Biomech Sports 1996;13:175-83

3. Brodowicz GR, Welsh R, Wallis J. Comparison of Stretching with Ice, Stretching with Heat, or Stretching Alone on Hamstring Flexibility. Journal of Athletic Training. Journal of Athletic Training 1996;31:324-7.

4. Bandy WD, Irion JM, Briggler M. The Effect of Time and Frequency of Static Stretching on Flexibility of the Hamstring Muscles. Phys Ther 1997;77:1090-6.

5. Anderson B, Burke ER. Scientific, medical, and practical aspects of stretching. Clin Sports Med 1991;10:63-86.

6. Magnusson SP. Passive properties of human skeletal muscle during stretch maneuvers: a review. Scand J Med Sci Sports 1998;8:65-77.

7. Kubo K, Kanehisa H, Kawakami Y, Fukunaga T. Influence of static stretching on viscoelastic properties of human tendon structures in vivo. J Appl Physiol 2001;90:520-7.

8. Lentell G, Hetherington T, Eagan J, Morgan M. The use of thermal agents to influence the effectiveness of a low-load prolonged stretch. J Orthop Sports Phys Ther 1992;16:200-7.

9. Taylor BF, Waring CA, Brashear TA. The effects of therapeutic application of heat or cold followed by static stretch on hamstring muscle length. J Orthop Sports Phys Ther 1995;21:283-6.

10. Usuba M, Miyanaga Y, Miyakawa S, Maeshima T, Shirasaki Y. Effect of Heat in Increasing the Range of Knee Motion After the Development of a Joint Contracture: An Experiment With an Animal Model. Arch Phys Med Rehabil 2006;87:247-53.

11. Burke DG, Holt LE, Rasmussen R, MacKinnon NC, Vossen JF, Pelham TW. Effects of Hot or Cold Wate Immersion and Modified Proprioceptive Neuromuscular Facilitation Flexibility Exercise on Hamstring Length. J Athl Train 2001;36:16-9.

12. Cornelius W, Jackson A. The effects of cryotherapy and PNF on hip extensor flexibility. Athl Train $J$ Natl Athl Train Assoc 1984;19:183-99.

13. Wilder RP, Greene JA, Winters KL, Long WB 3rd, Gubler K, Edlich RF. Physical fitness assessment: an update. J Long Term Eff Med Implants 2006;16:193-204.

14. Wells KF, Dillon KE. The sit and reach: a test of back and leg flexibility. Res Q 1952;23:115-8.

15. Holt LE, Pelham TW, Burke DG. Modifications to the Standard Sit-and-Reach Flexibility Protocol. J Athl Train 1999;34:43-7.

16. Williams PE, Goldspink G. Connective tissue changes in immobilized muscle. J Anat (London) 1984;138:342-50.

17. Prado LG, Makarenko I, Andresen C, Kruger M, Opitz CA, Linke WA. Isoform diversity of giant proteins in relation to passive and active contractile properties of rabbit skeletal muscles. J Gen Physiol 2005;126:461-80.

18. Gajdosik RL, Allred JD, Gabbert HL, Sonsteng BA. A stretching program increases the dynamic passive length and passive resistive properties of the calf muscle-tendon unit of unconditioned younger women. Eur J Appl Physiol 2007;99:449-54.

19. Cross KM, Worrell TW. Effects of a Static Stretching Program on the Incidence of Lower Extremity Musculotendinous Strains. Journal of Athletic Training 1999;34:11-4.

20. Thacker SB, Gilchrist J, Stroup DF, Kimsey CD Jr. The impact of stretching on sports injury risk: a systematic review of the literature. Med Sci Sports Exerc 2004;36:371-8.

21. Jayaraman RC, Reid RW, Foley JM, Prior BM, Dudley GA, Weingand KW, et al. MRI evaluation of topical heat and static stretching as therapeutic modalities for the treatment of eccentric exercise-induced muscle damage. Eur J Appl Physiol 2004;93:30-8.

22. Draper DO, Castro JL, Feland B, Schulthies S, Eggett D. Shortwave diathermy and prolonged stretching increase hamstring flexibility more than prolonged stretching alone. J Orthop Sports Phys Ther 2004;34:13-20.

23. Proulx Cl, Ducharme MB, Kenny GP. Effect of water temperature on cooling efficiency during hyperthermia in humans. J Appl Physiol 2003;94:1317-23.

24. Viveiros L, Polito MD, Simão R, Farinatti P. Respostas agudas imediatas e tardias da flexibilidade na extensão do ombro em relação ao número de séries e duração do alongamento. Rev Bras Med Esporte 2004;10:459-63.

25. Nyland J, Kocabey Y, Caborn DN. Sex differences in perceived importance of hamstring stretching among high school athletes. Percept Mot Skills 2004;99:3-11. 